\title{
Planeación de la Expansión de la Transmisión con Reforzamiento de la red a partir de la evaluación de la confiabilidad
}

\author{
Transmission Expansion Planning with Enhancement Network from
}

\author{
Reliability Assessment \\ Néstor González Cabrera ${ }^{1,2}$, L. Fernando Fuerte Ledezma ${ }^{1}$, Guillermo Gutiérrez Alcaraz ${ }^{1}$ \\ ${ }^{1}$ Instituto Tecnológico de Morelia, Programa de Graduados e Investigación en Ingeniería Eléctrica, Morelia, \\ Michoacán. \\ ${ }^{2}$ Instituto Tecnológico Superior de Irapuato, Departamento de Electromecánica, Irapuato, Guanajuato \\ nestor.gonzalez@itesi.edu.mx
}

\begin{abstract}
Resumen-Uno de los principales factores para la planeación de la transmisión se establece a partir de diversos criterios como la minimización de los costos de inversión, operación o ambos, además de criterios de confiabilidad en la generación, en el sistema de transmisión o en el sistema compuesto generación-transmisión. En este trabajo se resuelve el problema de expansión de la transmisión (PET) estático, el problema de expansión es formulado mediante Programación Entera Mixta (MIP, Mix Integer Programming). El problema se resuelve en dos fases secuenciales: la primera fase resuelve el PET mediante GAMS la segunda fase evalúa la confiabilidad del plan de expansión óptimo y se adicionan nuevas líneas a fin de reducir los índices de confiabilidad hasta un valor definido o cero. La principal aportación en este trabajo es la implementación en Matlab-GAMS para la solución al PET considerando la evaluación de la confiablidad. Para mostrar la efectividad de la metodología se utiliza el sistema de 6 nodos de Garver y el sistema escandinavo de 12 nodos.
\end{abstract}

Palabras clave- Confiabilidad, Expansión, Planeación, Transmisión,

Abstract-One of the main factors for transmission expansion is established from various criteria as the minimization of inversion, operation or both, criteria's of reliability in generation, in transmission system or the composite system. In this paper the static transmission expansion planning (TEP) is solved, the problem is formulated by Mix Integer Programming (MIP). And is solved in two sequential phases: first phase the TEP is solved by GAMS and the second phase assess the reliability of the optimum expansion plan and new lines are added with the finality of reduce the reliability indices to a defined value or zero. The principal contribution of this work is the implementation of the interface Matlab-GAMS to solve the TEP considering the reliability assessment. To show the effectiveness of the methodology 6 nodes Garver system and 12 nodes Scandinavian system are used. Key Word -Expansion, Planning, Transmission, Reliability

$$
\text { I. INTRODUCCIÓN }
$$

Los sistemas de transmisión permiten el transporte de energía eléctrica producida por generadores de distintas características a los centros de consumo. Por ello, las redes de transmisión se han desarrollado extensamente, dando lugar a la creación de sistemas eléctricos interconectados.

El problema de la expansión de la transmisión (PET), ha sido abordado desde la década de los 70's [1-5], consiste en determinar las líneas que deben ser adicionadas para satisfacer la demanda futura de manera segura y confiable, con el mínimo costo de inversión. El PET puede ser clasificado como estático o dinámico $[6,7-1,2]$. En el problema estático se planea un solo horizonte de tiempo, es decir se define inicialmente un horizonte de planificación y se obtiene una proyección de la demanda para este horizonte; la inversión total del proyecto de expansión se efectúa al inicio del periodo de planificación [8-3]. En el problema dinámico, el periodo de estudio se divide en subperíodos, en los cuales se resuelve un problema estático, y se determina en qué año se adhieren las líneas de transmisión [83].

La confiabilidad es uno de los criterios de mayor importancia en la operación de un sistema eléctrico de potencia, sin embargo, tener una red más confiable generalmente requiere mayores gastos de inversión, por lo que el problema conlleva a tener un conflicto entre el costo de inversión y una confiabilidad óptima..La confiabilidad se ha integrado como una restricción o como una función objetivo en el TEP, usando diversos criterios probabilísticos tales como Probabilidad de Pérdida de Carga (LOLP -Loss Of Load Probability), Pérdida de Carga Esperada (LOLE -Lost Of Load Expected), Energía Esperada No Suministrada (EENS -Expected Energy Not Supplied). El primer trabajo reportado con la integración de la confiabilidad fue registrado en los 80's [9], donde se propone una metodología cuantitativa para incorporar restricciones de confiabilidad a la expansión de la transmisión. El objetivo de la 
metodología propuesta es lograr un nivel de confiabilidad del sistema prescrito para los elementos que presentan sobrecarga. La sensibilidad de los índices de confiabilidad para las respectivas capacidades de los elementos de transmisión son calculados iterativamente hasta hallar el valor de confiabilidad prescrito. Una metodología que utiliza criterios probabilísticos es propuesta en [10-4] la cual determina un plan óptimo para la expansión de la transmisión usando criterios que minimizan los costos esperados. El índice utilizado es el LOLE y es determinado minimizando el valor total de la construcción y el costo de interrupción del suministro eléctrico. Otra metodología basada en criterios probabilísticos ha sido reportada en [11-5], en el cual se propone un proceso de selección para la captura de las mejores alternativas con la evaluación de los modelos de confiabilidad, a través del sistema jerárquico. Se enumeran los estados y se utiliza el método de Monte Carlo no secuencial. En [12-6] se propone una metodología cuantitativa para incorporar restricciones de confiabilidad a la expansión de la transmisión. El objetivo de la metodología propuesta es lograr un nivel de confiabilidad del sistema prescrito para los elementos que presentan sobrecarga. La sensibilidad de los índices de confiabilidad para las respectivas capacidades de los elementos de transmisión son calculados y son aumentados iterativamente hasta hallar el valor de confiabilidad prescrito. El método es capaz de identificar las capacidades óptimas en los cambios de generación y transmisión requeridos para conocer el índice antes mencionado. En [13-7], los autores proponen una nueva metodología para resolver el PET basado en técnicas de optimización meta heurísticas (colonia de hormigas). El PET incluye la solución con mínimo costos, teniendo en cuenta costos de inversión y confiabilidad, considerándose el valor de la confiabilidad como el costo de interrupción en el sistema (LOLC-Loss Of Load Cost). En [14-8] se propone un PET probabilístico para minimizar la suma de las inversiones, la demanda esperada no servida y la generación no servida. En este, se analizan probabilísticamente las contingencias utilizando la simulación de Monte Carlo con un modelo de flujos en DC. En [15] utilizan el método de la ruleta como proceso para la selección de las mejores alternativas de inversión basados en criterios probabilísticos de operación, la metodología propuesta propone: minimizar los costos de inversión, la demanda esperada no servida y la generación esperada no servida. En [169] proponen un modelo multi-objetivo capaz de evaluar la integración de distintas granjas de generación eólicas asociadas a la red de transmisión para la planeación del sistema eléctrico, los autores evalúan invertir en nuevas líneas tomando en cuenta el nivel jerárquico II del sistema de potencia (GeneracionTransmision) utilizando como principal indicador la EENS.

El principal aporte de este trabajo es la implementación en Matlab-GAMS para resolver el problema del PET considerando la Energía Nodal No Suministrada (ENNS) como índice para la toma de decisión de reforzamiento de la red. Se presentan dos casos de estudio utilizando sistemas eléctricos de potencia reportados en la literatura, el sistema de 6 nodos de Garver y el sistema escandinavo de 12 nodos. El resto del trabajo está organizado de la siguiente forma: en la siguiente sección se estudia la formulación del PET, posteriormente se estudia la confiabilidad del sistema eléctrico de potencia, la sección continua explica la metodología propuesta, continuando con los casos de estudio y finalmente se presentan las conclusiones.

\section{FORMULACION MATEMATICA DEL PET}

El objetivo del PET es minimizar los costos de inversión y los costos de operación mientras se satisface un conjunto de restricciones operativas. La formulación matemática se puede escribir de la siguiente manera:

$$
\begin{gathered}
\text { Min } C T=\sum_{j \in E^{+}}^{N L^{+}} \mathrm{CI}_{j}+\sum_{i=j}^{N G} C G_{i}\left(P G_{i}\right) \\
\text { Sujeto } a \quad C f+P G+P G V=D^{i} \\
f_{i j}=\frac{\left(E_{i j}^{0}+N_{i j}\right)\left(\theta_{i}-\theta_{j}\right)}{X_{i j}} \\
-f_{i j}^{M a x} \leq f_{i j} \leq f_{i j}^{\text {Max }} \\
P G_{i}^{M i n} \leq P G_{i} \leq P G_{i}^{M a x} \\
0 \leq P G V_{j} \leq P G V_{j}^{\text {Max }} \\
E_{i j}^{0} \leq\left(E_{i j}^{0}+N_{i j}\right) \leq N_{i j}^{M a x}
\end{gathered}
$$

donde son los costos de inversión de la nuevas líneas de transmisión, son los costos de operación asociado a la generación de potencia en el nodo $i$, es vector de demanda con elementos $D_{\_} j$ para cada estado de demanda $i$, es la reactancia de la línea que conecta los nodos i y j, es el número de nuevas líneas conectadas entre los nodos i y j, es el número de líneas existentes que conecta los nodos i y $\mathrm{j}$, el ángulo del voltaje complejo nodal en el nodo $i$, es el flujo de potencia activa a través de la línea que conecta los nodos i y j, es el flujo máximo de potencia activa a través de la línea que conecta los nodos i y $\mathrm{j}$, es la matriz de incidencia nodal, es el vector de los flujos con elementos , es la potencia generada en el nodo , es el vector de elementos, y son los límites de generación para cada generador, es el número máximo de líneas que se pueden añadir entre nodos i y j, PGV es el vector de generación virtual con elementos PGVj, es la generación virtual, es el límite superior de generación virtual, es el número de generadores existentes y es el número de nuevas líneas.

Los costos de operación de la generación térmica son de la forma cuadrática y está representada por:

$$
C G_{i}\left(P G_{i}\right)=a_{i}+b_{i} P G_{i}+c_{i}\left(P G_{i}\right)^{2}
$$

donde, y representan los coeficientes de los costos del generador i.

\section{EVALUACION DE LA CONFIABILIDAD}

Para la operación del sistema debe garantizarse el suministro continuo de energía. Para hacer el cálculo de cualquier índice 
probabilístico se necesita obtener las disponibilidades e indisponibilidades de los elementos a analizar, en este trabajo se realizaron solo análisis en los componentes de transmisión considerando la parte de generación $100 \%$ confiable.

La indisponibilidad $\left(U_{c}\right)$ del componente fallado es la división de las fallas anuales $\left(\lambda_{c}\right)$ del componente conectado en el nodo sobre las reparaciones anuales $\left(\mu_{c}\right)$ del elemento conectado en el nodo y es calculado a partir de [7-10]:

$$
U_{c}=\left(\frac{\lambda_{c}}{\mu_{c}}\right)
$$

La disponibilidad del componente $\left(A_{c}\right)$, puede calcularse como la resta de uno menos la indisponibilidad del elemento conectado al nodo con respecto al elemento fallado.

$$
A_{c}=1-U_{c}
$$

Posteriormente la probabilidad de ocurrencia de falla de los componentes es calculada a partir de:

$$
p_{j}=\prod_{c=1}^{b} U_{c} \prod_{c=b+1}^{N c} A_{c}
$$

donde $p_{j}$ es la probabilidad de ocurrencia del estado $j$, con $b$ elementos fallados y $N_{c}$ componentes independientes. El cálculo de la ENNS [18] para cada contingencia se obtiene de acuerdo con:

$$
E N N S_{i}=P G V_{i}^{j}
$$

donde $P G V_{i}^{j}$ es la representación de la ENS para aliviar la sobre carga debido a la contingencia $j$. La ENNS es simulada como un generador virtual con costos mayores a la de los generados existentes, que representa la ENS a los usuarios, y sólo se genera cuando no se cumple el balance de energía y se tengan que realizar cortes de carga en el nodo.

El cálculo de la Energía Nodal Esperada No Suministrada (ENENS-Expected Nodal Energy Not Supplied) para cada contingencia se obtiene mediante la siguiente expresión:

$$
E N E N S_{i}=E N N S_{i}^{j} \times p_{j}
$$

La Energía Esperada No Suministrada (EENS-Expected Energy Not Supplied) del sistema es la sumatoria de todas las ENENS para todos los estados posibles de orden N-1, es decir:

$$
E E N S=\sum_{i=1}^{N N} \sum_{j=1}^{N T E} E_{N N S S_{i}^{j}}
$$

donde $N N$ es el número de nodos del sistema, $N T E$ es el número total de estados o contingencias $\mathrm{N}-1$.

\section{METODOLOGIA PARA EVALUAR LA EXPANSION DE LA TRANSMISION ESTATICA}

La solución del PET considerando la ENNS se resuelve en forma secuencial, como se muestra en la figura 1. Inicialmente se cargan los datos (generación, líneas, demanda, proyectos de expansión) en Matlab, posteriormente se transfieren a GAMS y se resuelve el PET, una vez obtenida la solución, se escribe un archivo de salida en formato *.m en el cual se indican las nuevas líneas de transmisión, posteriormente la solución del PET es leída en Matlab y hace los cálculos de confiabilidad y si esta cumple con el criterio mínimo establecido se considera la solución ideal, si no está dentro de parámetros, Matlab se encarga de reforzar el sistema agregando una nueva línea de transmisión en la contingencia donde se genera la mayor ENNS, este proceso es iterativo y termina cuando el sistema está dentro de los parámetros establecidos.

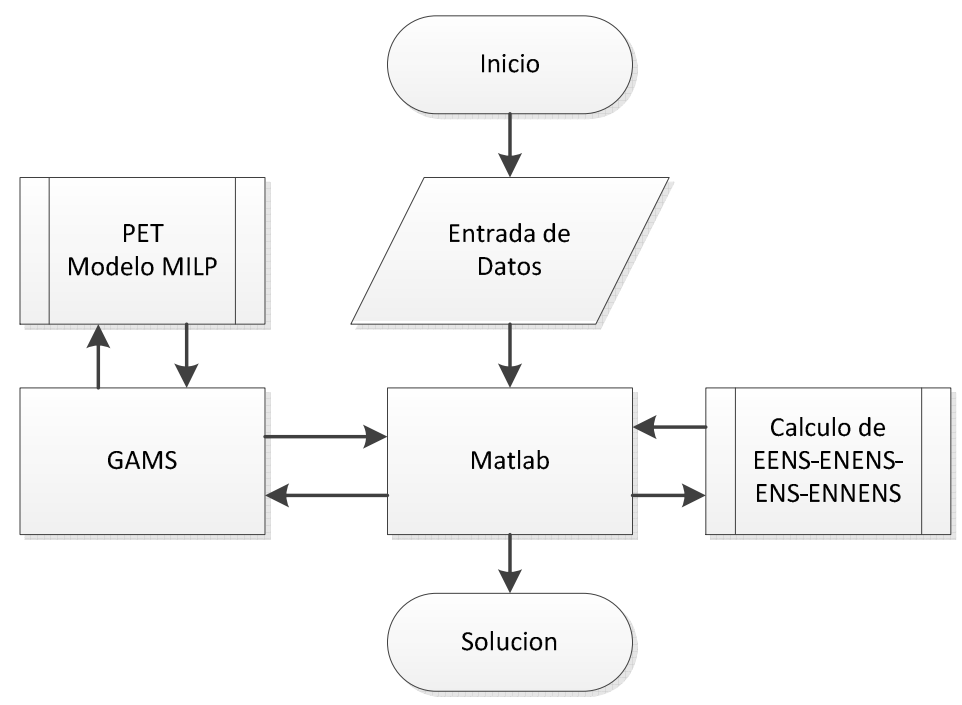

Figura 1. Modelo de Solución PET-Reforzamiento de red

A continuación se enumeran los pasos a seguir que muestran la metodología del PET-reforzamiento de la red:

Paso 1. Se introducen los datos del sistema y los proyectos de expansión (líneas candidatas)

Paso 2. Se resuelve el PET sin considerar el criterio de ENNS Paso 3. Matlab toma la nueva configuración, y mediante un proceso iterativo realiza N-1 contingencias para conocer los valores de ENNS y ENENS.

i. Se determina el número total de estados (N-1 contingencias)

ii. Se evalúa el primer estado (elimina una línea del sistema).

iii. Utilizando teoría de grafos se determina si la nueva la configuración presentan nodos aislados (islas). Si existen islas continúan de lo contrario ir a v.

iv. Se determina el número de islas. En cada isla se verifica en esta si existe generación, si es el caso se realiza un estudio de Flujos de Potencia Óptimos. Se revisa el valor obtenido de los generados virtuales siendo este el valor de ENNS. Sí no existe generación se contabiliza el valor de la demanda en cada nodo siendo esta el valor de ENNS. Ir a vi.

v. Se realiza un estudio de Flujos de Potencia Óptimos. Se revisa el valor obtenido de los generadores virtuales siendo este el valor de ENNS. 
vi. $\quad$ Se verifica si este es el último estado. Si es el último estado entonces se procede al paso vii, de lo contrario se evalúa el siguiente estado y se procede al paso iii

vii. $\quad$ Se suman la ENNS de todo el sistema.

Paso 4. Se compara la ENNS con un valor predefinido, si la ENNS es menor que dicho valor se termina el PET de lo contrario se procede al paso 5

Paso 5. Se identifica el nodo con mayor ENNS.

Paso 6. Se agrega una nueva línea entre los nodos con mayor ENNS.

Paso 7. Se agregan los costos de inversión de las nuevas líneas. Se determinan los costos de operación (Mediante la Curva de duración de carga para 3 periodos de tiempo, Carga Máxima, Media y Mínima, de todo el año) con las nuevas líneas.

Paso 8. Se vuelve a comparar las ENNS hasta obtener el valor deseado.

El diagrama de flujo de la metodología propuesta se presenta en la figura 2.

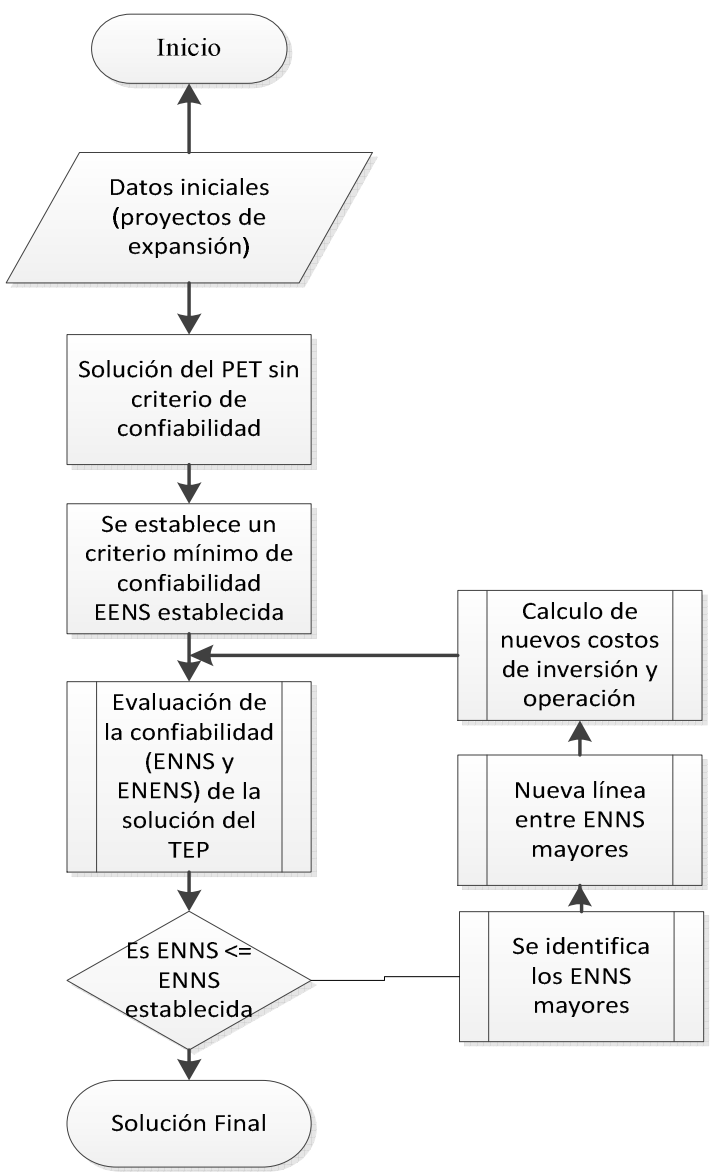

Figura 2. Diagrama de flujos del PET-Reforzamiento de red.

\section{CASO DE ESTUDIO}

\section{A) CASO 1}

En esta sección se presentan dos casos de estudio. El primer caso utiliza la red de 6 nodos de Garver [1]. Se considera un horizonte de planificación de 10 años y se estima que la demanda crecerá alrededor del $400 \%$. La figura 3 muestra el diagrama unifilar de la red de 6 nodos, en la cual se muestra el sistema al final del año 10. Se observa que el nodo 6 es un nodo de generación aislado, resultado de un proyecto de expansión de generación. Adicionalmente, se consideran 40 líneas candidatas para la expansión de la transmisión.

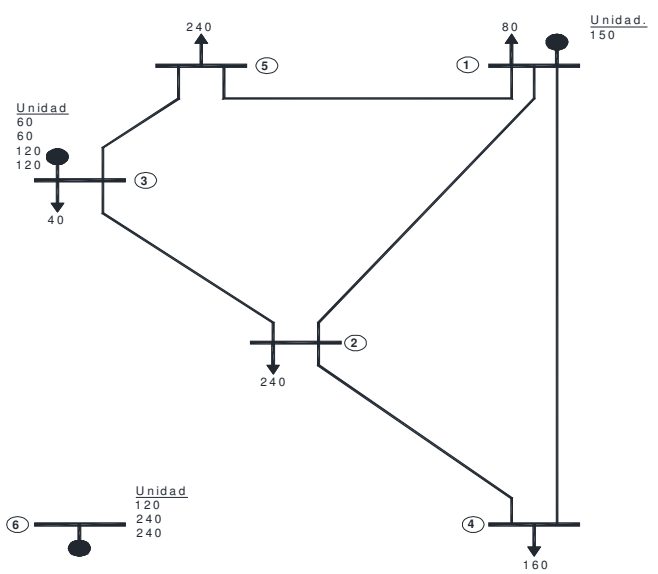

Figura 3. Condiciones futuras del Sistema Garver de 6 nodos para el año 10.

La tabla I presenta las características de las 40 líneas candidatas para la expansión de la transmisión.

TABLA I: PROYECTOS DE LAS LÍNEAS PROPUESTAS.

\begin{tabular}{|c|c|c|c|c|}
\hline $\begin{array}{c}\text { Número de } \\
\text { Líneas }\end{array}$ & $\begin{array}{c}\text { Nodo de Envío- } \\
\text { Recepción }\end{array}$ & $\begin{array}{c}\mathrm{X} \\
(\mathrm{pu})\end{array}$ & $\begin{array}{c}\text { Potencia Máxima } \\
(\mathrm{MW})\end{array}$ & $\begin{array}{c}\text { Costo } \\
\text { (MUSD \$) }\end{array}$ \\
\hline 2 & $1-2$ & 0,40 & 100 & 40 \\
\hline 3 & $1-3$ & 0,38 & 100 & 38 \\
\hline 2 & $1-4$ & 0,60 & 80 & 60 \\
\hline 2 & $1-5$ & 0,20 & 100 & 60 \\
\hline 3 & $2-6$ & 0,68 & 70 & 68 \\
\hline 2 & $2-3$ & 0,20 & 100 & 20 \\
\hline 2 & $2-4$ & 0,40 & 100 & 40 \\
\hline 3 & $2-5$ & 0,31 & 100 & 31 \\
\hline 4 & $2-6$ & 0,30 & 100 & 30 \\
\hline 3 & $3-4$ & 0,59 & 82 & 59 \\
\hline 2 & $3-5$ & 0,20 & 100 & 20 \\
\hline 3 & $3-6$ & 0,48 & 100 & 48 \\
\hline 3 & $4-5$ & 0,63 & 75 & 63 \\
\hline 3 & $4-6$ & 0,30 & 100 & 30 \\
\hline 3 & $5-6$ & 0,61 & 78 & 61 \\
\hline
\end{tabular}

En la figura 4 se muestra la curva de duración de carga (CDC) del año 10. Para la elaboración de esta curva se tomó el patrón de demanda que se presenta en [19]. La CDC se agrupa en tres escenarios de demanda: demanda máxima, 1513 horas, demanda media, 4465 horas, y demanda mínima, 2782 horas. 


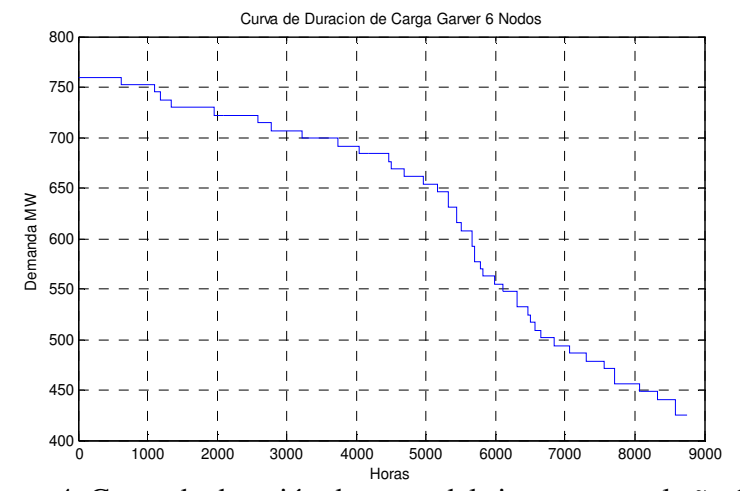

En la tabla II se observa la descomposición anual de la demanda del sistema. En la descomposición de la demanda se presenta la demanda desde el año 1, para los tres periodos de tiempo, y esta va aumentando un $32 \%$ aproximadamente por año hasta llegar al año 10 .

Figura 4. Curva de duración de carga del sistema para el año 10.

TABLA II. DESCOMPOSICIÓN DE LA DEMANDA ANUAL (MW) DEL SISTEMA.

\begin{tabular}{|c|c|c|c|c|c|c|c|c|c|c|}
\hline Año & 1 & 2 & 3 & 4 & 5 & 6 & 7 & 8 & 9 & 10 \\
\hline Máxima & 62,7 & 82,6 & 109,3 & 144,4 & 190 & 250,8 & 330,6 & 436,1 & 575,7 & 760 \\
\hline Media & 50,4 & 66,4 & 87,8 & 116,1 & 152,7 & 201,6 & 265,7 & 350,4 & 462,7 & 610,8 \\
\hline Mínima & 41,3 & 54,5 & 71,9 & 95,1 & 125,2 & 165,2 & 217,8 & 287,3 & 379,3 & 500,7 \\
\hline
\end{tabular}

La figura 5 muestra la solución del TEP sin considerar ningún criterio de confiabilidad.

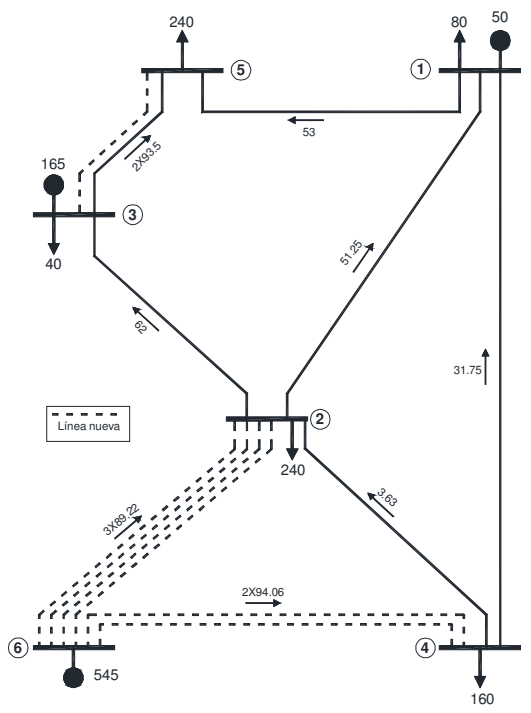

Figura 5. Configuración óptima del sistema de 6 nodos sin criterio de confiabilidad.

Las líneas punteadas representan las nuevas líneas a instalarse. 7 nuevas líneas son necesarias para acomodar la demanda futura.

Las líneas resultantes son: una línea entre los nodos 3 y 5 , cuatro líneas que conecta los nodos 2 y 6 , y dos líneas más que conectan los nodos 6 y 4 . Este resultado es el mismo al reportado [1].

Evaluando la confiabilidad (o seguridad) de la configuración optima al TEP, se obtiene la ENNS y la ENNS total (ver tabla III).

TABLA III. ENNS POR CONTINGENCIAS (MW/HR) DEL PET. \begin{tabular}{|l|l}
\hline Línea & Nodo \\
\hline
\end{tabular}

\begin{tabular}{|c|c|c|c|c|c|}
\cline { 1 - 1 } & 1 & 2 & 3 & 4 & 5 \\
\hline $1-2$ & 26,4 & 0 & 15,6 & 3,7 & 34 \\
\hline $1-4$ & 4,8 & 4,8 & 4,8 & 0 & 4,8 \\
\hline $1-5$ & 0 & 0 & 7,8 & 0 & 62,5 \\
\hline $2-3$ & 0 & 0 & 35,1 & 0 & 35,1 \\
\hline $2-4$ & 0 & 0 & 0 & 0 & 0 \\
\hline $3-5$ & 0 & 0 & 0 & 2,2 & 103,7 \\
\hline $3-5$ & 0 & 0 & 0 & 2,2 & 103,7 \\
\hline $2-6$ & 0 & 180,6 & 0 & 0 & 0 \\
\hline $2-6$ & 0 & 180,6 & 0 & 0 & 0 \\
\hline $2-6$ & 0 & 180,6 & 0 & 0 & 0 \\
\hline $2-6$ & 0 & 180,6 & 0 & 0 & 0 \\
\hline $4-6$ & 0 & 0 & 0 & 215,6 & 0 \\
\hline $4-6$ & 0 & 0 & 0 & 215,6 & 0 \\
\hline Total & 31,2 & 727,2 & 63,4 & 439,3 & 343,9 \\
\hline
\end{tabular}

De la tabla III se muestra que el nodo 4 presenta la mayor ENNS, derivado de la pérdida de la línea 4-6, de tal forma que si se desea reducir la ENNS es necesario adicionar una línea entre los nodos 4 y 6 . El nodo 6 no es representado en la tabla debido a que solo es nodo de generación y no existe demanda en este.

La tabla IV muestra la ENNS y la ENENS de la solución del PET.

TABLA IV. ENENS (MW/HR) DEL PET.

\begin{tabular}{|c|l|l|l|l|l|}
\hline \multirow{2}{*}{ Línea } & \multicolumn{5}{|c|}{ Nodo } \\
\cline { 2 - 6 } & 1 & \multicolumn{1}{|c|}{2} & \multicolumn{1}{|c|}{4} & \multicolumn{1}{c|}{5} \\
\hline $1-2$ & 0,091 & 0 & 0,053 & 0,012 & 0,116 \\
\hline $1-4$ & 0,023 & 0,023 & 0,023 & 0 & 0,023 \\
\hline $1-5$ & 0 & 0 & 0,012 & 0 & 0,106 \\
\hline $2-3$ & 0 & 0 & 0,059 & 0 & 0,059 \\
\hline $2-4$ & 0 & 0 & 0 & 0 & 0 \\
\hline $3-5$ & 0 & 0 & 0 &, 003 & 0,178 \\
\hline $3-5$ & 0 & 0 & 0 & 0,003 & 0,178 \\
\hline $2-6$ & 0 & 0,464 & 0 & 0 & 0 \\
\hline $2-6$ & 0 & 0,464 & 0 & 0 & 0 \\
\hline $2-6$ & 0 & 0,464 & 0 & 0 & 0 \\
\hline $2-6$ & 0 & 0,464 & 0 & 0 & 0 \\
\hline $4-6$ & 0 & 0 & 0 & 0,054 & 0 \\
\hline $4-6$ & 0 & 0 & 0 & 0,054 & 0 \\
\hline Total & 0,114 & 1,879 & 0,148 & 0,127 & 0,661 \\
\hline
\end{tabular}


En la tabla V se presenta la EENS anualizada resultado del PET, es decir sin reforzamiento de la red, de donde se puede apreciar como para el escenario de demanda máxima y media existe EENS.

TABLA V. EENS ANUALIZADA (GW/HR).

\begin{tabular}{|l|c|c|c|c|c|c|c|c|c|c|}
\hline Año & 1 & 2 & 3 & 4 & 5 & 6 & 7 & 8 & 9 & 10 \\
\hline Máxima & 0 & 0 & 0 & 0 & 0 & 0 & 0 & 0 & 0,14 & 5,19 \\
\hline Media & 0 & 0 & 0 & 0 & 0 & 0 & 0 & 0 & 0 & 2,38 \\
\hline Mínima & 0 & 0 & 0 & 0 & 0 & 0 & 0 & 0 & 0 & 0 \\
\hline
\end{tabular}

Los costos totales de operación, en Valor Presente con una tasa de interés del $10 \%$ anual, son del orden de \$27662490,7. En este trabajo no se consideran variaciones en los precios de los combustibles a lo largo del periodo de análisis

Considerando que se agrega una nueva línea entre los nodos 4 y 6 a fin de reducir la ENNS, la tabla VI presenta las nuevas ENNS.

\begin{tabular}{|c|c|c|c|c|c|}
\hline \multirow{2}{*}{ Línea } & \multicolumn{5}{|c|}{ Nodo } \\
\cline { 2 - 6 } & 1 & 2 & 3 & 4 & 5 \\
\hline $1-2$ & 0 & 0 & 15,62 & 0 & 29,68 \\
\hline $1-4$ & 0 & 0 & 0 & 0 & 0 \\
\hline $1-5$ & 0 & 0 & 7,81 & 0 & 62,48 \\
\hline $2-3$ & 0 & 0 & 35,15 & 0 & 35,15 \\
\hline $2-4$ & 0 & 0 & 0 & 0 & 0 \\
\hline $3-5$ & 0 & 0 & 0 & 0 & 98,69 \\
\hline $3-5$ & 0 & 0 & 0 & 0 & 98,69 \\
\hline $2-6$ & 0 & 117,78 & 0 & 0 & 0 \\
\hline $2-6$ & 0 & 117,78 & 0 & 0 & 0 \\
\hline $2-6$ & 0 & 117,78 & 0 & 0 & 0 \\
\hline $2-6$ & 0 & 117,78 & 0 & 0 & 0 \\
\hline $4-6$ & 0 & 0 & 0 & 0 & 0 \\
\hline $4-6$ & 0 & 0 & 0 & 0 & 0 \\
\hline Total & 0 & 471,13 & 58,58 & 0 & 324,68 \\
\hline
\end{tabular}

Tabla VI. ENNS por contingencias (MW) del reforzamiento de la red.

Comparando la solución de la tabla VI con la de la tabla IV, se puede observar claramente como al agregar nuevas líneas en los nodos con mayor ENNS se logra una reducción de ENNS. Esto se puede apreciar aún más al observar los valores de ENENS reportados en la tabla VII, en algunos nodos llegó a cero y en otros, como en el 2 se redujo en un $25 \%$.

\section{TABLA VII. ENENS POR CONTINGENCIA (MW/HR)} DEL REFORZAMIENTO DE LA RED.

\begin{tabular}{|c|c|c|c|c|c|}
\hline \multirow{2}{*}{ Línea } & \multicolumn{5}{|c|}{ Nodo } \\
\cline { 2 - 6 } & 1 & 2 & 3 & 4 & 5 \\
\hline $1-2$ & 0 & 0 & 0,05 & 0 & 0,10 \\
\hline $1-4$ & 0 & 0 & 0 & 0 & 0 \\
\hline $1-5$ & 0 & 0 & 0,01 & 0 & 0,11 \\
\hline $2-3$ & 0 & 0 & 0,06 & 0 & 0,06 \\
\hline $2-4$ & 0 & 0 & 0 & 0 & 0 \\
\hline $3-5$ & 0 & 0 & 0 & 0 & 0,17 \\
\hline $3-5$ & 0 & 0 & 0 & 0 & 0,17 \\
\hline $2-6$ & 0 & 0,30 & 0 & 0 & 0 \\
\hline $2-6$ & 0 & 0,30 & 0 & 0 & 0 \\
\hline $2-6$ & 0 & 0,30 & 0 & 0 & 0 \\
\hline $2-6$ & 0 & 0,30 & 0 & 0 & 0 \\
\hline $4-6$ & 0 & 0 & 0 & 0 & 0 \\
\hline $4-6$ & 0 & 0 & 0 & 0 & 0 \\
\hline Total & 0 & 1,21 & 0,12 & 0 & 0,60 \\
\hline
\end{tabular}

La figura 6 resume gráficamente el proceso de adición de nuevas línea en el sistema, a fin de reducir a cero la ENNS.

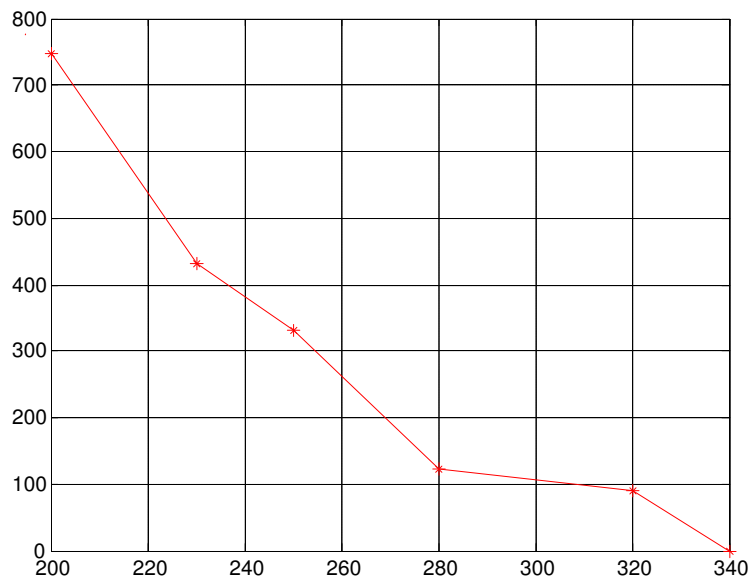

Figura 6. Costos de inversión para mejorar la confiabilidad del sistema.

Caso 2

En la figura 7 se presenta el diagrama del sistema escandinavo de 12 nodos [20], el periodo de planeación para este sistema es de 10 años, se muestran las 4 unidades de generación y las demandas en los nodos.

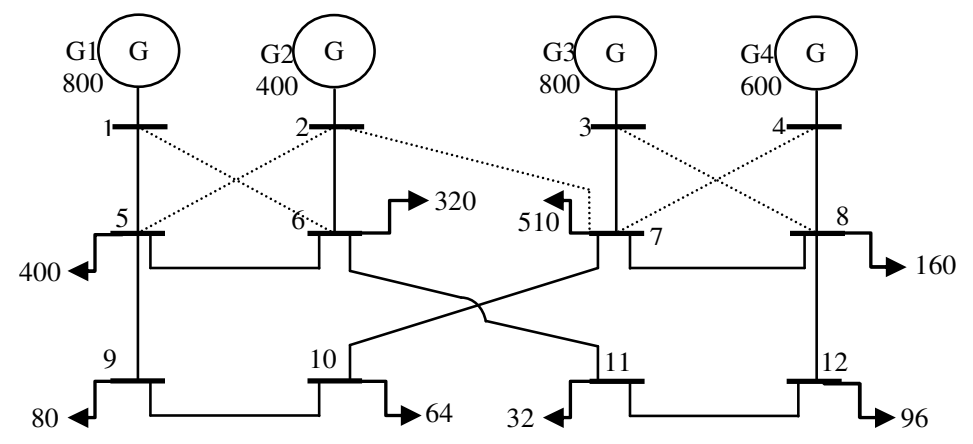


Figura 7. Diagrama del sistema de potencia de 12 nodos

Se tienen 6 nuevas líneas candidatas, con capacidad de 300 MW, en el caso de las líneas 3-8 una se marca como cable alambre y tiene una resistencia y capacitancia de la mitad de capacidad que la otra línea y diversos costos, además se pueden hacer reforzamientos en las líneas originales del sistema, pero se solo se puede considerar 1 línea máxima como reforzamiento, como se muestra en la tabla VIII.

TABLA VIII. DATOS DE LAS LÍNEAS DEL SISTEMA DE 12 NODOS.

\begin{tabular}{|c|c|c|c|c|}
\hline $\begin{array}{c}\text { Número de } \\
\text { Líneas }\end{array}$ & $\begin{array}{c}\text { Nodo de Envío- } \\
\text { Recepción }\end{array}$ & $\begin{array}{c}\mathrm{X} \\
(\mathrm{pu})\end{array}$ & $\begin{array}{c}\text { Potencia Máxima } \\
(\mathrm{MW})\end{array}$ & $\begin{array}{c}\text { Costo } \\
\text { MUSD \$) }\end{array}$ \\
\hline 1 & $1-6$ & 0,0592 & 300 & 1,5 \\
\hline 1 & $2-5$ & 0,0592 & 300 & 1,5 \\
\hline 1 & $2-7$ & 0,1656 & 300 & 7,5 \\
\hline 1 & $3-8$ & 0,1656 & 300 & 2,5 \\
\hline 1 & $3-8^{*}$ & 0,0552 & 300 & 7,5 \\
\hline 1 & $4-7$ & 0,2376 & 300 & 3,5 \\
\hline 1 & $5-6$ & 0,0592 & 300 & 10 \\
\hline 1 & $7-8$ & 0,1980 & 300 & 10 \\
\hline 1 & $1-5$ & 0,0118 & 600 & 10 \\
\hline 1 & $2-6$ & 0,0118 & 600 & 10 \\
\hline 1 & $3-7$ & 0,0118 & 600 & 10 \\
\hline 1 & $4-8$ & 0,0118 & 600 & 10 \\
\hline 1 & $5-9$ & 0,0864 & 300 & 10 \\
\hline 1 & $7-10$ & 0,0864 & 300 & 10 \\
\hline 1 & $9-10$ & 0,0864 & 300 & 10 \\
\hline 1 & $6-11$ & 0,1093 & 300 & 10 \\
\hline 1 & $8-12$ & 0,1093 & 300 & 10 \\
\hline 1 & $11-12$ & 0,1093 & 300 & 10 \\
\hline
\end{tabular}

En la figura 8 se presenta la curva de duración de carga del sistema de 12 nodos, para obtener esta curva se utilizó un procedimiento similar al caso 1, la demanda Máxima presenta 1183 horas, la Media 5315 horas y la Mínima 2262 horas anuales.

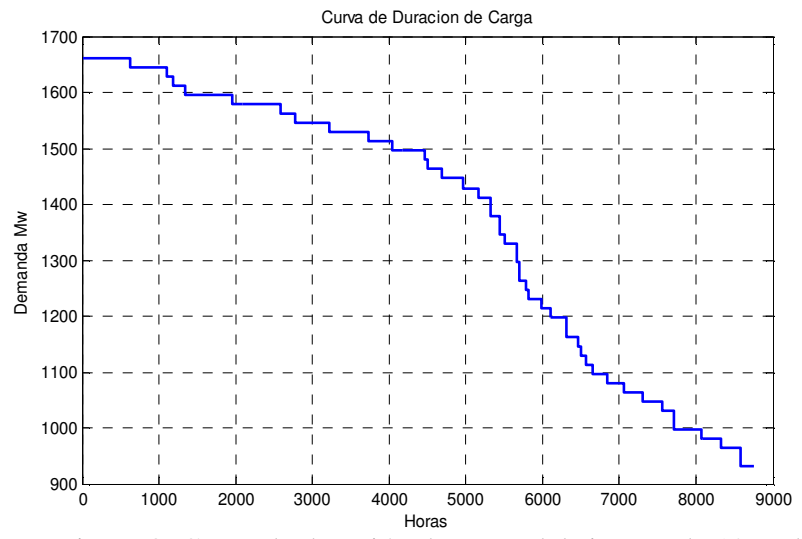

Figura 8. Curva de duración de carga del sistema de 12 nodos.

La tabla IX muestra la descomposición de la demanda anual para el sistema de 12 nodos.

TABLA IX. DESCOMPOSICIÓN DE LA DEMANDA ANUAL MW DEL SISTEMA DE 12 NODOS.

\begin{tabular}{|c|c|c|c|c|c|c|c|c|c|c|}
\hline Año & $\mathbf{1}$ & $\mathbf{2}$ & $\mathbf{3}$ & $\mathbf{4}$ & $\mathbf{5}$ & $\mathbf{6}$ & $\mathbf{7}$ & $\mathbf{8}$ & $\mathbf{9}$ & $\mathbf{1 0}$ \\
\hline Máxima & 137,1 & 180,7 & 238,9 & 315,8 & 415,5 & 548,5 & 723 & 953,6 & 1259 & 1662 \\
\hline Media & 110,2 & 145,3 & 192 & 253,8 & 333,9 & 440,8 & 581 & 766,4 & 1011,8 & 1335,7 \\
\hline Mínima & 90,3 & 119,1 & 157,4 & 208,1 & 273,8 & 361,4 & 476,3 & 628,3 & 829,5 & 1095,1 \\
\hline
\end{tabular}

En la tabla X se muestran las diversas ENNS hechas en el sistema cuando se aplica análisis de contingencias de n-1. TABLA X. ENNS (MW) EN EL SISTEMA ANTES DEL
PET

\begin{tabular}{|c|c|c|c|c|c|c|c|c|}
\hline Línea & N5 & N6 & N7 & N8 & N9 & N10 & N11 & N12 \\
\hline $\mathbf{1 - 5}$ & 201 & 145 & 1236 & 1642 & 883 & 127 & 174 & 875 \\
\hline $\mathbf{2 - 6}$ & & 0 & 0 & 9843 & 2958 & 3343 & 251 & 2553 \\
\hline $\mathbf{3 - 7}$ & 105 & 962 & 265 & 378 & 98 & 110 & 188 & 851 \\
\hline $\mathbf{4 - 8}$ & 0 & 0 & 0 & 7018 & 2107 & 2381 & 251 & 182 \\
\hline $\mathbf{5 - 9}$ & 0 & 0 & 0 & 0 & 0 & 0 & 0 & 0 \\
\hline $\mathbf{5 - 6}$ & 0 & 0 & 0 & 0 & 0 & 0 & 0 & 0 \\
\hline $\mathbf{6 - 1 1}$ & 0 & 0 & 0 & 127 & 3819 & 4326 & 251 & 330 \\
\hline $\mathbf{7 - 8}$ & 0 & 0 & 0 & 127 & 3819 & 4326 & 251 & 330 \\
\hline $\mathbf{7 - 1 0}$ & 0 & 0 & 0 & 0 & 0 & 0 & 0 & 0 \\
\hline $\mathbf{8 - 1 2}$ & 0 & 0 & 0 & 127 & 3819 & 4326 & 251 & 330 \\
\hline $\mathbf{9 - 1 0}$ & 0 & 0 & 0 & 127 & 3819 & 4326 & 251 & 33 \\
\hline
\end{tabular}


De la tabla $\mathrm{X}$ se observa que en el nodo 10 presenta la mayor ENNS ante las salidas de las líneas que conectan al nodo directamente. Posteriormente en la tabla XI se muestra la ENNS por contingencia para la solución del PET.

TABLA XI. ENNS (MW/HR) POR CONTINGENCIA DE LA SOLUCIÓN DEL PET.

\begin{tabular}{|c|c|c|c|c|c|c|c|c|}
\hline Línea & N5 & N6 & N7 & N8 & N9 & N10 & N11 & N12 \\
\hline $1-5$ & 86 & 63 & 47 & 70 & 39 & 55 & 70 & 39 \\
\hline $2-6$ & 0 & 0 & 0 & 39 & 125 & 141 & 109 & 109 \\
\hline $3-7$ & 39 & 39 & 109 & 16 & 3 & 47 & 117 & 31 \\
\hline $4-8$ & 0 & 0 & 0 & 23 & 856 & 101 & 101 & 94 \\
\hline $5-9$ & 0 & 0 & 0 & 0 & 0 & 0 & 0 & 0 \\
\hline $5-6$ & 0 & 0 & 0 & 0 & 0 & 0 & 0 & 0 \\
\hline
\end{tabular}

\begin{tabular}{|c|c|c|c|c|c|c|c|c|}
\hline $6-11$ & 0 & 0 & 0 & 63 & 187 & 211 & 12 & 164 \\
\hline $7-8$ & 0 & 0 & 0 & 47 & 141 & 164 & 94 & 125 \\
\hline $7-10$ & 0 & 0 & 0 & 0 & 0 & 0 & 0 & 0 \\
\hline $8-12$ & 0 & 0 & 0 & 63 & 187 & 211 & 125 & 164 \\
\hline $9-10$ & 0 & 0 & 0 & 63 & 203 & 242 & 133 & 180 \\
\hline Total & 125 & 102 & 156 & 384 & 1741 & 1172 & 761 & 906 \\
\hline
\end{tabular}

La figura 9 presenta la solución del PET sin criterio de confiabilidad, el cual arroja como solución óptima la inserción de dos líneas en el sistema, una que conecta los nodos 1 y 6 y otra que conecta los nodos 3 y 8 .

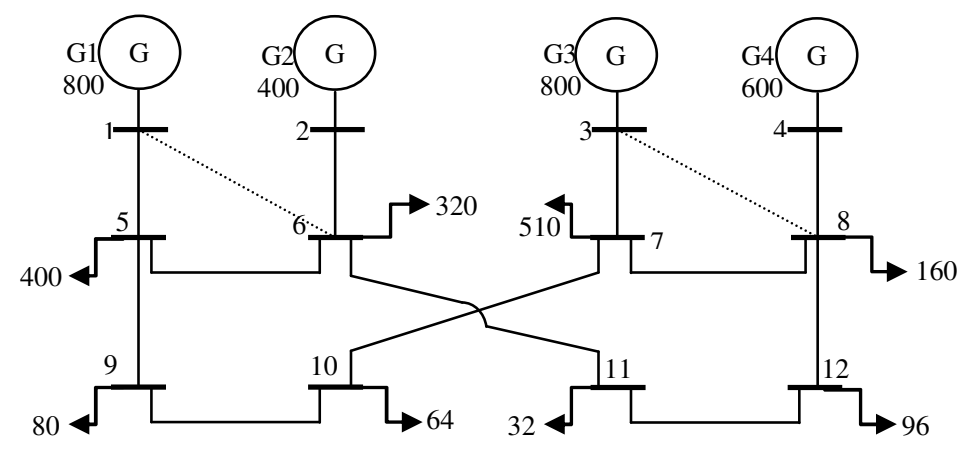

Figura 9. Diagrama del sistema de potencia de 12 nodos después de la solución del PET.

En la tabla XII muestra los nodos y líneas que presentan ENNS, una vez obtenida la solución del modelo de expansión de la transmisión.

TABLA XII. ENNS (MW/HR) SIN REFORZAMIENTO DE RED.

\begin{tabular}{|c|c|c|c|c|c|c|c|c|}
\hline Línea & N5 & N6 & N7 & N8 & N9 & N10 & N11 & N12 \\
\hline $1-5$ & 177 & 0 & 0 & 0 & 0 & 0 & 0 & 0 \\
\hline $2-6$ & 0 & 0 & 0 & 0 & 0 & 0 & 0 & 0 \\
\hline $3-7$ & 0 & 0 & 357 & 0 & 0 & 64 & 0 & 0 \\
\hline
\end{tabular}

Se aprecia que aunque la planeación se expande el sistema de transmisión con 2 líneas más, existen cortes de carga en algunos nodos para contingencias de $\mathrm{N}-1$. La tabla XIII muestra los nodos donde se presenta ENNS en el sistema después de la expansión de la transmisión.
TABLA XIII. ENNS POR CONTINGENCIA (MW/HR) DEL REFORZAMIENTO DE LA RED.

\begin{tabular}{|c|c|c|c|c|c|c|c|c|}
\hline Línea & N5 & N6 & N7 & N8 & N9 & N10 & N11 & N12 \\
\hline $\mathbf{1 - 5}$ & 0,07 & 0 & 0 & 0 & 0 & 0 & 0 & 0 \\
\hline $\mathbf{2 - 6}$ & 0 & 0 & 0 & 0 & 0 & 0 & 0 & 0 \\
\hline $\mathbf{3 - 7}$ & 0 & 0 & 1,48 & 0 & 0 & 0,02 & 0 & 0 \\
\hline
\end{tabular}

La tabla XIV muestra la EENS anualizada del sistema observando que en el año 10 presenta cortes de carga.

TABLA XIV. EENS ANUALIZADA DEL SISTEMA DE 12 NODOS (MW/HR).

\begin{tabular}{|l|r|r|r|r|r|r|r|r|r|r|r|}
\hline Año & 1 & \multicolumn{1}{|c|}{2} & 3 & 4 & 5 & 6 & 7 & 8 & 9 & \multicolumn{1}{|c|}{} \\
\hline Máxima & 0 & 0 & 0 & 0 & 0 & 0 & 0 & 0 & 0 & 39,5 \\
\hline Media & 0 & 0 & 0 & 0 & 0 & 0 & 0 & 0 & 0 & 0 \\
\hline Mínima & 0 & 0 & 0 & 0 & 0 & 0 & 0 & 0 & 0 & 0 \\
\hline
\end{tabular}

Por último, en la figura 10 se muestra la solución final si se requiere una ENNS de cero, línea adicional entre los nodos 3 y 7. 


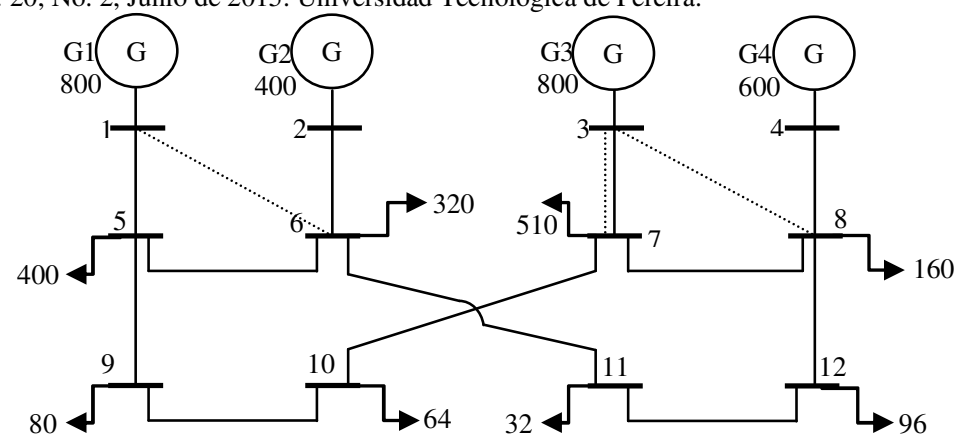

Figura 10. Diagrama del sistema de potencia de 12 nodos con el reforzamiento de la red.

En la figura 11 se presenta el análisis de costos de inversión, en el primer punto se puede apreciar que aplicando la solución del PET resulta una inversión inicial de \$ 4 millones, pero al hacer el análisis de confiabilidad, se observó que todavía existían nodos con cortes de carga. Una vez encontrada la solución para tener la ENS en cero con el reforzamiento de la red en 3-7 el costo final de inversión es de \$14 millones.

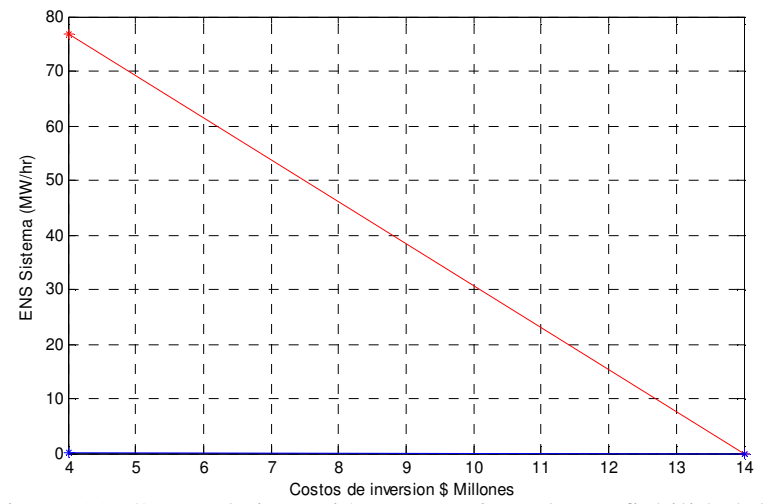

Figura 11. Costos de inversión para mejorar la confiabilidad del sistema de 12 nodos.

En la figura 12 se aprecia que para este caso el reforzamiento de la red está por debajo de la solución del PET sin confiabilidad en el horario de demanda media, es decir el reforzamiento hecho en los nodos 3-7 contribuye significativamente en el análisis de confiabilidad para el proyecto a 10 años incluso estando en los primeros años con valores cercanos a 0 EENS en el horario de demanda Máxima y en horario de demanda Media es hasta el año 6 donde se presentan cortes de carga cuando se hacen análisis de confiabilidad. También se aprecia que para ambas soluciones no se tiene EENS en el horario valle ya que este horario está considerado alrededor del $60 \%$ de la demanda máxima, debido a esto no presenta problemas de confiabilidad.

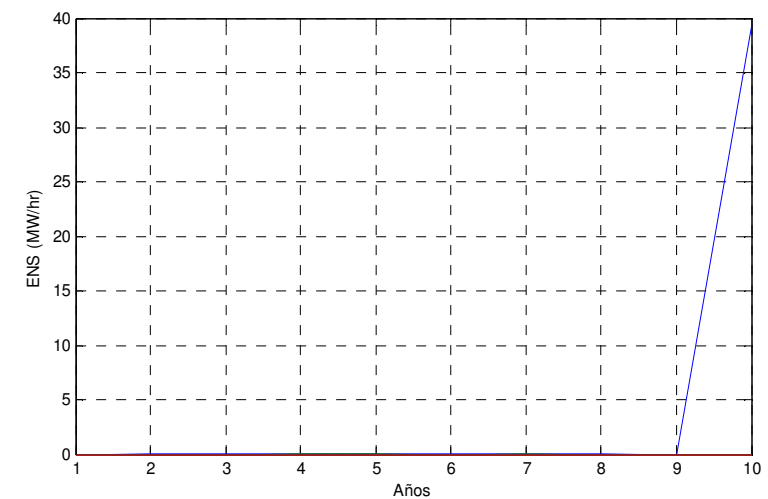

Figura 12. Curvas de EENS anual con reforzamiento de la red.

El EENS es utilizado como criterio de decisión para la adición de nuevas líneas, sin embargo se pueden utilizar otros tipos de criterios de confiabilidad como el LOLP o LOLE.

\section{Discusión}

La inversión para obtener un mejor servicio que sea seguro, confiable y continuo es el objetivo de cualquier sistema eléctrico, debido a eso, se realizan diversos estudios de planificación y operación, en los cuales se hace énfasis en los costos. En la tabla XV se presenta el Valor Presente Neto (VPN) para la operación del sistema de 12 nodos, en esta se observan las dos diversas soluciones en los distintos horarios. En la segunda columna se observa una disminución en los costos totales de operación e inversión, debido a la operación del sistema, ya que al haber mayor número de líneas permite que se despachen los generadores de mayor capacidad y menor costo.

TABLA XV. COSTOS DE OPERACIÓN EN VPN.

\begin{tabular}{|l|c|c|}
\hline & $\begin{array}{c}\text { TEP } \\
(\$ T)\end{array}$ & $\begin{array}{c}\text { Reforzamiento } \\
(\$ \mathrm{~T})\end{array}$ \\
\hline Máxima & 1,032 & 0,633 \\
\hline Media & 4,29 & 1641 \\
\hline Mínima & 4,29 & 3362 \\
\hline Costos Totales & 9,61 & 5,64 \\
\hline
\end{tabular}

Dentro de la parte operativa se aprecia como la solución de reforzamiento de red disminuye el costo de operación del 
sistema, sobre todo porque en algunos casos tener un sistema más mallado permite a los generadores lejanos más económicos poder transmitir toda la capacidad de generación tanto en la parte real como en la reactiva, requiriendo menos cortes de carga ante alguna contingencia.

Aunque esta metodología se utilizó para redes relativamente pequeñas, también puede ser utilizada en grandes redes, para las cuales el tiempo de solución sería mayor. Una de las ventajas de este método es que los encargados de la planificación pueden tener una referencia para posibles reforzamientos en la red, si es que los usuarios exigieran mayor confiabilidad en sus zonas o si se presentaran problemas de operación debido a una EENS mayor a la esperada o un incremento en la demanda al estimado por el pronóstico.

\section{CONCLUSIONES}

En este trabajo se presentó la formulación y solución al PET considerando el criterio de ENNS. El PET se resuelve en forma secuencial mediante Matlab-GAMS. Se utilizó el sistema de 6 nodos de Garver y un sistema de 12 nodos, obteniendo resultados similares en comparación con la referencia revisada. Se puedo observar en ambos caso que si se requiere de un sistema de transmisión robusto requerirá una mayor inversión. Además los costos de operación después del PET son mayores en comparación con los costos de operación después del reforzamiento de la red, ya que se reduce el congestionamiento en la red a partir de la inserción de más líneas después del PET.

\section{REFERENCIAS}

[1] L. L. Garver, Transmission network estimation using linear programming, IEEE Trans. Power Apparat. Syst., 1970.

[2] A. Monticelli, A. Santos, M. V. F. Pereira, S. H. Cunha, B. J. Parker and J. C. G. Praca, Interactive transmission network planning using a least-effort criterion, IEEE Trans. Power Syst., 1982.

[3] R. Villasana, L. L. Garver, and S. J. Salon, Transmission network planning using linear programming, "IEEE Trans. Power Apparat. Syst., 1985.

[4] M. A. Farrag and M. M. El-Metwally, New method for transmission planning using mixed-integer programming, IEE Proc., 1988

[5] R. Romero and A. Monticelli, A Hierarchical decomposition approach for transmission network expansion planning, IEEE Trans. Power Syst., 1994.

[6] Ricardo Andres Bolaños Ocampo, Planteamiento multiobjetivo de la planeación de la transmision considerando seguridad e incertidumbre de la demanda. Diciembre, 2009

[7] Andrés Inzunza B., Jaime Larraín. "Criterio N-1 y Automatismos de Control en la Expansión de los Sistemas de Transmisión". Tesis de maestria, Pontificia universidad católica de chile, 2011

[8] Augusto Cesar Rueda Medina. planeamiento de la expansión de sistemas de transmisión con incertidumbre en la demanda e incorporando perdidas usando búsqueda tabú. Tesis de Maestria Universidad Tecnológica de Pereira, 2008

[9] T.A.M. Sharaf and G.J. Berg, "Reliability optimization for Transmission Expansion Planning", IEEE Transactions on
Power Apparatus and Systems, Vol. PAS-101, No. 7 July 1982

[10] Ferris M. MATLAB and GAMS: Interfacing Optimization and Visualization Software. Available at: http://www.cs.wisc.edu/math-prog/matlab.html

[11] Armando M. Leite da Silva, Leandro S. Rezende, Luiz A. da Fonseca Manso, Leonidas C. de Resende, "Reliability worth applied to transmission expansion planning based on ant colony system", International Journal of Electrical Power \& Energy Systems Volume 32, Issue 10, December 2010, Pages 1077-1084

[12]B.RAlizadeh, S.Jadid,. "Reliability constrained coordination of generation and transmission expansion planning in power systems using mixed integer programming”, IET Gener. Transm. Distrib., 2011, Vol. 5, Iss. 9, pp. 948-960

[13] J. Choi, T.D. Mount, R.J. Thomas and R. Billinton , "Probabilistic reliability criterion for planning transmission system expansions", IEE Proc.-Gener. Transm. Distrib., Vol. 153, No. 6, November 2006

[14] L.A.F. Manso, A.M. Leite da Silva, "Probabilistic criteria for power system expansion planning", Electric Power Systems Research vol. 69, pp.51-58 , December2004

[15]Neeraj Gupta, Rajiv Shekhar, Prem Kumar Kalra, "Congestion management based roulette wheel simulation for optimal capacity selection: Probabilistic transmission expansion planning", Electric Power Systems Research vol. 43, pp.1259-1266, (July 2012).

[16] M. S. Javadia, M. Sanieia,, H. Rajabimashhadi, G. Gutiérrez-Alcaraz, "A Multi-objective Expansion Planning Approach: Distant Wind Farms and Limited Energy Resources Integration", IET Renewable Power Generation, pp. 17 47, August 2013.

[!7] Nestor Gonzalez Cabrera, "Mercado de Energia con multiple clases en confiabilidad considerando preferencias de usuarios finales", Tesis de Maestria, Instituto Tecnologico de Morelia, Agosto 2010

[18]P. Wang, Y. Ding and Y. Xiao, "Technique to evaluate nodal reliability indices and nodal prices of restructured power systems", IEE Proc.-Gener. Transm. Distrib., Vol. 152, No. 3, May 2005

[19] "IEEE-Reliability Test System" Reported prepared by the Reliability Test System Task Force of the Application of Probability Methods Subcommittee, IEEE Trans. Power Systems, Vol. 14,pp.1010-1021, August 1999.

[20] Aleksa B.Babic, Andrija T. Saric, Aleksandar Rankovic, "Transmission expansion planning based on locational marginal prices and ellipsoidal approximation of uncertainties", Eelctrical Power and Energyy Systems, Vol. 53, pp. 175-183 